\title{
Demonstration of the sensitivity of the Smith predictor to parameter uncertainties using stability diagrams
}

\author{
David Hajdu • Tamas Insperger
}

Received: 17 September 2014 / Revised: 25 November 2014 / Accepted: 29 November 2014

(C) Springer-Verlag Berlin Heidelberg 2014

\begin{abstract}
Time-domain representation of the original Smith predictor is presented for systems with feedback delay. Sensitivity to parameter uncertainties is analyzed for a marginally stable, for an asymptotically stable and for an unstable second-order plant. A series of stability diagrams are constructed using the D-subdivision method and Stepan's formulas. Transition to the stability diagrams subjected to delayed state feedback is established. It is demonstrated that the Smith predictor is sensitive to infinitesimal parameter mismatches for the marginally stable plant. It is shown that the Smith predictor can stabilize unstable plants for some extremely detuned internal model parameters.
\end{abstract}

Keywords Feedback delay $\cdot$ Smith predictor .

Time-domain representation $\cdot$ Parameter mismatch $\cdot$ Stability

\section{Introduction}

Control of unstable systems with feedback delay is a challenging problem in engineering and science [1-4]. Time delay in the feedback loop itself can cause instabilities and oscillations; examples include car following traffic models [5], crane payload stabilization [6], flutter instability [7] and consensus protocols [8]. Compensation of feedback delay is therefore a highly important task in engineering.

The Smith predictor [9] is probably the best known technique to overcome the destabilizing effect of feedback delays

D. Hajdu $\cdot$ T. Insperger $(\bowtie)$

Department of Applied Mechanics, Budapest University

of Technology and Economics, Budapest, Hungary

e-mail: insperger@mm.bme.hu

D. Hajdu

e-mail: hajdu@mm.bme.hu in control systems. It is known that state prediction is a fundamental concept for systems with feedback delay [10]. The main idea behind predictive controllers is that the feedback delay is eliminated from the control loop by using a prediction of the actual state based on an internal model of the plant. Since its publication, several types of modifications of the Smith predictor have been developed [10-16], most of them are related to the so-called finite spectrum assignment [17-19].

The Smith predictor is usually represented in frequency domain either by its block diagram or by its transfer function $[15,20,21]$. In the current paper, the time-domain representation of the Smith predictor is also given. We assume throughout the paper that the delayed state is fully available and thus there is no need for an observer.

A main concern about prediction-based controllers is that they require knowledge about the plant and the feedback delay. The slightest mismatch between the internal model used for the prediction and the actual system may destabilize the closed-loop system. The conditions for practical stability (i.e. the preservation of stability for infinitesimal modeling mismatches) for the Smith predictor was given in [22] and for some special cases in [23-25]. The effect of delay mismatches was investigated in [20]. It is known that in case of a proper but not strictly proper delay-free transfer function, the sensitivity to infinitesimal parameter mismatches can be explained by the discontinuity of the associated difference equation $[20,26]$. In this paper, we consider a system with a strictly proper delay-free transfer function and show that sensitivity to infinitesimal parameter mismatches can still occur for a marginally stable plant. Note that this sensitivity is of different nature from those analyzed in $[20,26]$. The sensitivity presented here is due to the preservation of the robustness of the plant to parameter uncertainties. Here, we visualize the sensitivity of the Smith predictor to parameter mismatches by 
constructing stability diagrams for a marginally stable, for an asymptotically stable and for an unstable second-order plant. These diagrams are the counterpart of the stability diagrams of second-order systems (such as the inverted pendulum) subjected to delayed state feedback, which serve as a reference for stabilizing unstable systems [27-29]. Transition between the Smith predictor and delayed state feedback is illustrated by a series of stability diagrams.

It is a general view that the original Smith predictor can be applied only to stable open-loop systems. In this paper, we show that this statement is not true if the internal model does not match the actual system. We use the above secondorder plant to demonstrate that an unstable open-loop system can be stabilized by the Smith predictor in case of large parameter mismatches. It should be emphasized that the proposed stabilization of unstable systems by the original Smith predictor has limited practical relevance. There are different modifications of the Smith predictor, which are designed to stabilize unstable systems, such as the modified Smith predictor $[11,15]$, the unified Smith predictor [16] or the finite spectrum assignment $[18,19]$.

The structure of the article is as follows. In Sect. 2, frequency-domain and time-domain representations of the Smith predictor are given. Stability analysis of a secondorder system subjected to the Smith predictor is presented in Sect. 3. Then the effect of parameter uncertainties on the stability is analyzed for a marginally stable plant in Sect. 4, for an asymptotically stable plant in Sect. 5 and for an unstable plant in Sects. 6 and 7 discusses some properties of the modifications of the Smith predictor. The results are concluded in Sect. 8.

\section{The Smith predictor}

The Smith predictor was developed in the frequency domain [9]. Time-domain representations are rarely discussed in the literature (see Eq. (2.45) in the book [10] for an exception). In this section, the time-domain equations of the Smith predictor are presented based on its block diagram.

\subsection{Frequency-domain representation}

The block diagram of the Smith predictor is shown in Fig. 1. As mentioned in the Introduction, the point of the Smith predictor is that the feedback delay is eliminated from the control loop using a prediction of the actual state based on an internal model of the plant. Let us denote the transfer function of the plant by $P(s)$, the transfer function of the plant used by the internal model by $\tilde{P}(s)$, the transfer function of the primary controller by $C(s)$, the actual feedback delay by $\tau$ and the delay used by the internal model by $\tilde{\tau}$. In practice,

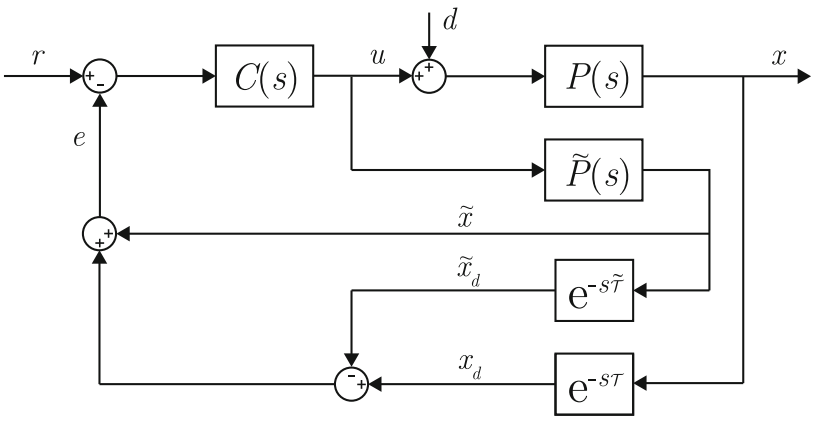

Fig. 1 The block diagram of the Smith predictor

the internal model is not perfectly accurate, therefore $P(s) \neq$ $\tilde{P}(s)$ and $\tau \neq \tilde{\tau}$.

The transfer function from the plant input disturbance $d$ to the output $x$ can be given as

$W_{d x}(s)=\frac{P(s)\left(1+C(s) \tilde{P}(s)-C(s) \tilde{P}(s) \mathrm{e}^{-\tilde{\tau} s}\right)}{1+C(s) \tilde{P}(s)-C(s) \tilde{P}(s) \mathrm{e}^{-\tilde{\tau} s}+C(s) P(s) \mathrm{e}^{-\tau s}}$.

If the plant and the controller are factorized as

$P(s)=\frac{B_{1}(s)}{A_{1}(s)}, \quad \tilde{P}(s)=\frac{\tilde{B}_{1}(s)}{\tilde{A}_{1}(s)}, \quad C(s)=\frac{B_{2}(s)}{A_{2}(s)}$,

then the transfer function reads

$$
\begin{aligned}
W_{d x}(s)= & \left(\tilde{A}_{1}(s) A_{2}(s) B_{1}(s)+B_{1}(s) \tilde{B}_{1}(s) B_{2}(s)\left(1-\mathrm{e}^{-\tilde{\tau} s}\right)\right) \\
& \times\left(A_{1}(s) A_{2}(s) \tilde{A}_{1}(s)+A_{1}(s) B_{2}(s) \tilde{B}_{1}(s)\left(1-\mathrm{e}^{-\tilde{\tau} s}\right)\right. \\
& \left.+\tilde{A}_{1}(s) B_{1}(s) B_{2}(s) \mathrm{e}^{-\tau s}\right)^{-1}
\end{aligned}
$$

Clearly, if $\tilde{A}_{1}(s)=A_{1}(s)$, then the poles of the open-loop system [which are the zeros of $\left.A_{1}(s)\right]$ are the poles of the closed-loop system, too. This deduction is often used to conclude that the Smith predictor cannot stabilize an unstable plant. However, if $\tilde{A}_{1}(s) \neq A_{1}(s)$ then this conclusion is not valid. Actually, while the original Smith predictor can stabilize stable or unstable plants in case of perfect matching of the internal model and the plant, it has been shown that it can be very sensitive to infinitesimal variations for unstable plants [14]. In Sect. 6 we show that in case of parameter mismatches, the Smith predictor can actually stabilize an unstable plant.

\subsection{Time-domain representation}

Time-domain representation of the Smith predictor in case of a state feedback primary controller can be written as 
$\dot{\mathbf{x}}(t)=\mathbf{A} \mathbf{x}(t)+\mathbf{B u}(t)$,

$\dot{\tilde{\mathbf{x}}}(t)=\tilde{\mathbf{A}} \tilde{\mathbf{x}}(t)+\tilde{\mathbf{B}} \mathbf{u}(t)$,

$\mathbf{u}(t)=\mathbf{K}(\mathbf{x}(t-\tau)-\tilde{\mathbf{x}}(t-\tilde{\tau})+\tilde{\mathbf{x}}(t))$,

where $\mathbf{x} \in \mathbb{R}^{n}$ is the vector of actual state variables, $\tilde{\mathbf{x}} \in \mathbb{R}^{n}$ is the auxiliary vector of predicted state variables, $\mathbf{A}$ and $\tilde{\mathbf{A}}$ are the actual and the model state matrices, $\mathbf{B}$ and $\tilde{\mathbf{B}}$ are the actual and the model input matrices and matrix $\mathbf{K}$ contains the control gains. Without loss of generality it can be assumed that the reference input $r$ is zero [if $r$ is not zero then the variational system around the reference input $r$ has the form of Eqs. (4)-(6)]. Equation (4) describes the actual system while Eq. (5) corresponds to the internal model. The corresponding control law can be given in integral form as

$$
\begin{aligned}
\mathbf{u}(t)= & \mathbf{K}\left(\mathbf{x}(t-\tau)-\int_{0}^{t-\tilde{\tau}} \mathrm{e}^{\tilde{\mathbf{A}}(t-\tilde{\tau}-\theta)} \tilde{\mathbf{B}} \mathbf{u}(\theta) \mathrm{d} \theta\right. \\
& \left.+\int_{0}^{t} \mathrm{e}^{\tilde{\mathbf{A}}(t-\theta)} \tilde{\mathrm{B}} \mathrm{u}(\theta) \mathrm{d} \theta\right) .
\end{aligned}
$$

Thus, the control law involves integrals of the control input over the interval $[0, t]$. The closed-loop system can be described by a system of retarded functional differential equations (RFDEs) with two delays ( $\tau$ and $\tilde{\tau}$ ) as

$$
\begin{aligned}
& \dot{\mathbf{x}}(t)=\mathbf{A} \mathbf{x}(t)+\mathbf{B} \mathbf{K}(\mathbf{x}(t-\tau)-\tilde{\mathbf{x}}(t-\tilde{\tau})+\tilde{\mathbf{x}}(t)), \\
& \dot{\tilde{\mathbf{x}}}(t)=\tilde{\mathbf{A}} \tilde{x}(t)+\tilde{\mathbf{B}} \mathbf{K}(\mathbf{x}(t-\tau)-\tilde{\mathbf{x}}(t-\tilde{\tau})+\tilde{\mathbf{x}}(t)) .
\end{aligned}
$$

The characteristic equation is

$\operatorname{det}\left(\begin{array}{cc}s \mathbf{I}-\mathbf{A}-\mathbf{B} \mathbf{K} \mathrm{e}^{-\tau s} & -\mathbf{B K}\left(1-\mathrm{e}^{-\tilde{\tau} s}\right) \\ -\tilde{\mathbf{B}} \mathbf{K} \mathrm{e}^{-\tau s} & s \mathbf{I}-\tilde{\mathbf{A}}-\tilde{\mathbf{B}} \mathbf{K}\left(1-\mathrm{e}^{-\tilde{\tau} s}\right)\end{array}\right)=0$.

It can be seen that the closed-loop system subjected to the Smith predictor is equivalent to a delayed state feedback system with an augmented state vector of dimension double that of the open-loop system.

\section{Stability analysis for a second-order system}

We consider the system (4)-(6) with

$$
\mathbf{A}=\left(\begin{array}{rr}
0 & 1 \\
-a & 0
\end{array}\right), \quad \tilde{\mathbf{A}}=\left(\begin{array}{cc}
0 & 1 \\
-\tilde{a} & 0
\end{array}\right), \quad \mathbf{B}=\tilde{\mathbf{B}}=\left(\begin{array}{c}
0 \\
-1
\end{array}\right)
$$

and $\mathbf{K}=\left(\begin{array}{ll}p & d\end{array}\right)$, where $a$ is a system parameter, $\tilde{a}$ is the estimated system parameter used by the internal model, $p$ and $d$ are the control gains. This system describes the behavior of a second-order system with $p$ and $d$ being the proportional and the derivative control gains. If $a>0$ then the open-loop system is marginally stable, if $a<0$ then it is unstable.

\subsection{Special case: delayed state feedback}

If $\tilde{\mathbf{A}}=\mathbf{0}$ and $\tilde{\mathbf{B}}=\mathbf{0}$ then the predicted state is constant in time and Eq. (8) gives the delayed state feedback

$\dot{\mathbf{x}}(t)=\mathbf{A x}(t)+\mathbf{B K} \mathbf{x}(t-\tau)$.

The associated characteristic function is

$D(s)=s^{2}+a+(p+s d) \mathrm{e}^{-\tau s}$.

The stability diagram of this special case can be constructed by the D-subdivision method [30]. The D-curves, which are associated with pure imaginary characteristic exponents of the form $s=\mathrm{i} \omega$, can be given in the parametric form

if $\omega=0: \quad p=-a, \quad d \in \mathbb{R}$,

if $\omega \neq 0:\left\{\begin{array}{l}p=\left(\omega^{2}-a\right) \cos (\omega \tau) \\ d=\frac{\omega^{2}-a}{\omega} \sin (\omega \tau)\end{array}\right.$.

The number of unstable characteristic exponents in the domains separated by the D-curves can be determined using Stepan's formulas (see Eqs. (2.17) and (2.19) in [30]). The corresponding stability diagrams are shown in Fig. 2 for a stable and for an unstable open-loop system with $\tau=1$. It is known that the system cannot be stabilized if $a<a_{\text {crit }}=$ $-2 / \tau^{2}$ (see e.g., [29] or [30]).

\subsection{General case: the Smith predictor}

The stability diagram for a Smith predictor can be determined with the same procedure as used for delayed state feedback. Based on Eq. (10), the characteristic function of the system described by Eqs. (4)-(6) is

$$
\begin{aligned}
D(s)= & \left(s^{2}+\tilde{a}\right)\left(s^{2}+a\right)+(p+s d)\left(\left(s^{2}+a\right)\right. \\
& \left.+\left(s^{2}+\tilde{a}\right) \mathrm{e}^{-\tau s}-\left(s^{2}+a\right) \mathrm{e}^{-\tilde{\tau} s}\right) .
\end{aligned}
$$

Application of the D-subdivision method gives the D-curves in the following form. If $\omega=0$, then

$p=-a, \quad d \in \mathbb{R}$. 
Stable open-loop system

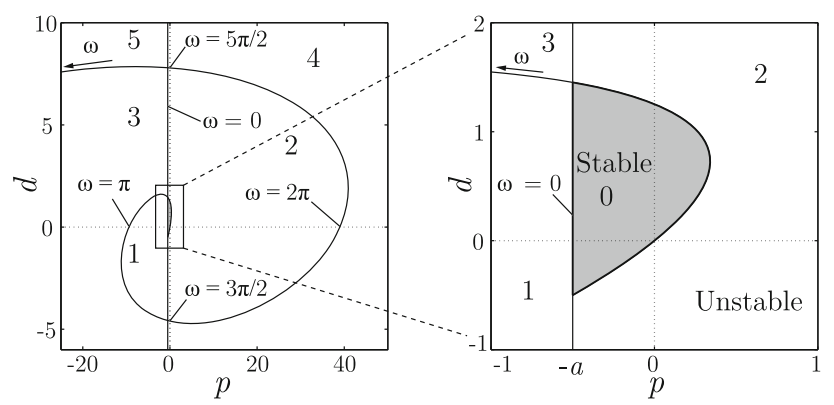

Unstable open-loop system

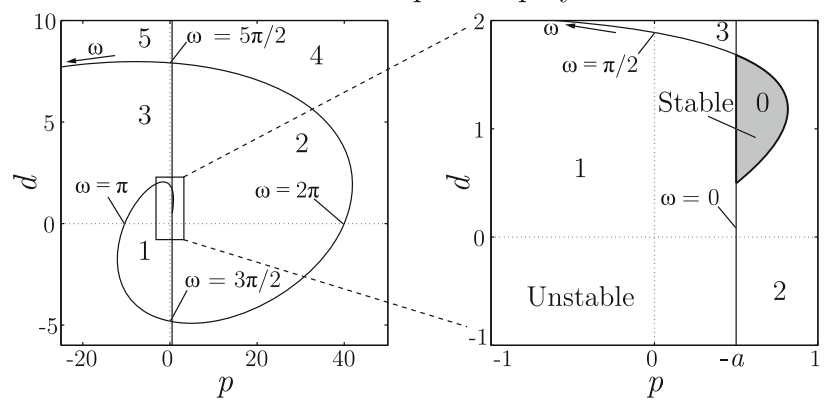

Fig. 2 Stability diagrams with the number of the unstable characteristic exponents for a marginally stable open-loop system with $a=0.5$ (top) and for an unstable open-loop system with $a=-0.5$ (bottom) subjected to delayed state feedback with feedback delay $\tau=1$. Regions of asymptotic stability are indicated by gray shading

This result is similar to that obtained for a delayed state feedback controller. If $\omega \neq 0$, then

$$
\begin{aligned}
p= & \left(( a - \omega ^ { 2 } ) ( \tilde { a } - \omega ^ { 2 } ) \left(\left(-\tilde{a}+\omega^{2}\right) \cos (\omega \tau)\right.\right. \\
& \left.\left.+\left(a-\omega^{2}\right)(-1+\cos (\omega \tilde{\tau}))\right)\right) /\left(2 a^{2}+\tilde{a}^{2}-4 a \omega^{2}\right. \\
& -2 \tilde{a} \omega^{2}+3 \omega^{4}+2\left(a-\omega^{2}\right)\left(\tilde{a}-\omega^{2}\right) \cos (\omega \tau) \\
& +2\left(a-\omega^{2}\right)\left(-\tilde{a}+\omega^{2}\right) \cos (\omega(\tau-\tilde{\tau}))-2 a^{2} \cos (\omega \tilde{\tau}) \\
& \left.+4 a \omega^{2} \cos (\omega \tilde{\tau})-2 \omega^{4} \cos (\omega \tilde{\tau})\right), \\
d= & \left(( a - \omega ^ { 2 } ) ( \tilde { a } - \omega ^ { 2 } ) \left(\left(-\tilde{a}+\omega^{2}\right) \sin (\omega \tau)\right.\right. \\
& \left.\left.+\left(a-\omega^{2}\right) \sin (\omega \tilde{\tau})\right)\right) /\left(\omega \left(2 a^{2}+\tilde{a}^{2}-4 a \omega^{2}\right.\right. \\
& -2 \tilde{a} \omega^{2}+3 \omega^{4}+2\left(a-\omega^{2}\right)\left(\tilde{a}-\omega^{2}\right) \cos (\omega \tau) \\
& +2\left(a-\omega^{2}\right)\left(-\tilde{a}+\omega^{2}\right) \cos (\omega(\tau-\tilde{\tau}))-2 a^{2} \cos (\omega \tilde{\tau}) \\
& \left.\left.+4 a \omega^{2} \cos (\omega \tilde{\tau})-2 \omega^{4} \cos (\omega \tilde{\tau})\right)\right) .
\end{aligned}
$$

Again, the number of unstable characteristic exponents can be determined using Stepan's formulas [30].

A sample stability diagram is shown in Fig. 3 for two different cases: when the system parameter $a$ is underestimated by the internal model ( $\tilde{a}<a$, top panels) and when the system parameter $a$ is overestimated ( $\tilde{a}>a$, bottom panels).
Underestimated parameter

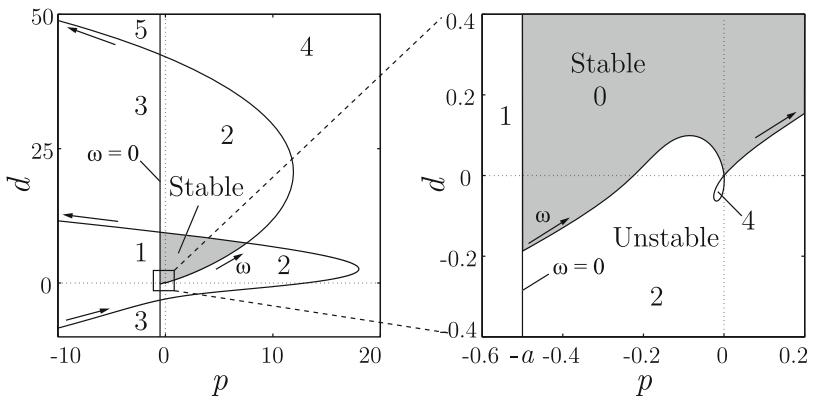

Overestimated parameter

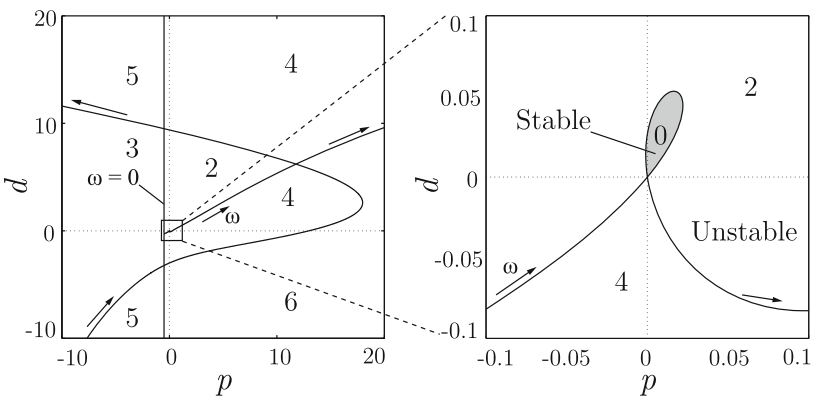

Fig. 3 Stability diagrams with the number of the unstable characteristic exponents for a marginally stable open-loop system subjected to the Smith predictor with $a=0.5, \tau=1, \tilde{\tau}=0.5 \tau$ for $\tilde{a}=0.8 a$ (top) and $\tilde{a}=1.2 a$ (bottom). Regions of asymptotic stability are indicated by gray shading

It can be seen that the stability diagram for the two cases are completely different. In the case of system parameter overestimation, the stable domain is reduced to a small loop attached to the origin $(p, d)=(0,0)$.

\section{Sensitivity analysis for a marginally stable plant}

Here we investigate the sensitivity to parameter mismatches for a marginally stable plant $(a>0)$. A series of stability diagrams with the number of unstable characteristic exponents are presented in Fig. 4 for different mismatches of the system parameter and the feedback delay. These diagrams can be considered projections of the 4-dimensional stability chart in the parameter space $(p, d, \tilde{a}, \tilde{\tau})$. For the ideal case, when $\tilde{a}=a$ and $\tilde{\tau}=\tau$, the stability boundaries are given by $p>-a$ and $d>0$, which corresponds to the stability condition for the delay-free system. In case of $\tilde{a}=a$, the rightmost characteristic exponents are purely imaginary, consequently, the system is marginally stable (see striped gray regions in Fig. 4). This property is inherited from the open-loop system [31]. However, if $\tilde{a} \neq a$ then the system becomes asymptotically stable (see gray shading regions in Fig. 4). It can be seen that stability properties change radically if $\tilde{a}>a$. Even the slightest overestimation of the system parameter can destabilize the system. Slight underestimation of the system 


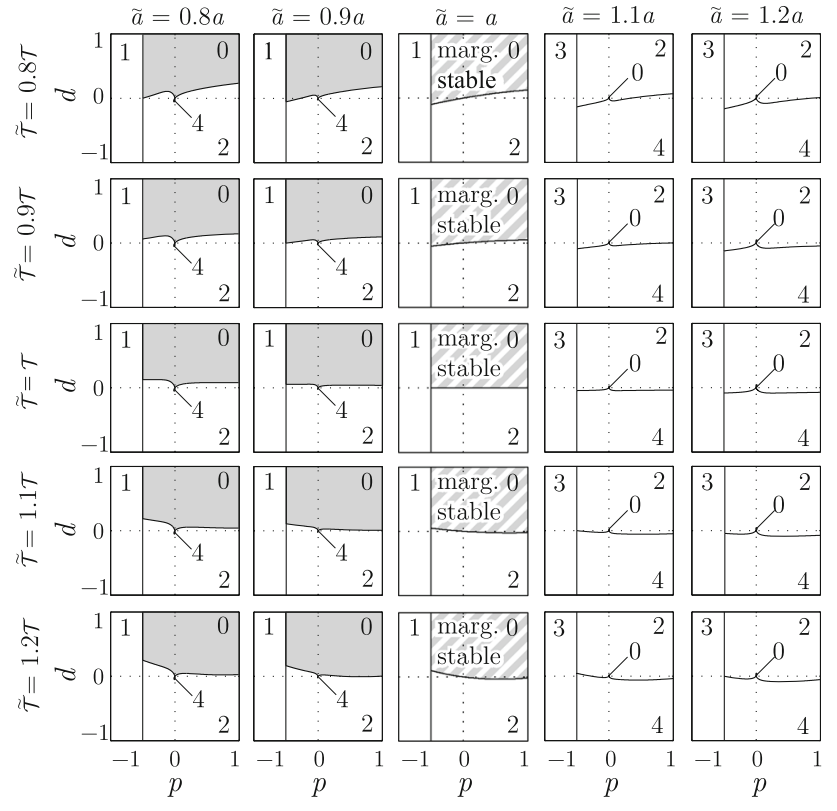

Fig. 4 Stability diagrams with the number of the unstable characteristic exponents for small parameter mismatches $(a=0.5, \tau=1)$. Regions of asymptotic stability and marginal stability are indicated by solid gray shading and by striped gray shading, respectively

parameter, however, does not affect the stability picture. The effect of the uncertainty in the time delay is smaller than that of the system parameter. In general, mismatches in the delay cause a slight reduction of the stable domains. Note that the D-curve $p=-a$ does not change for any system parameter mismatch.

Figure 5 shows the transition of the stability diagram for small mismatches of the estimated system parameter $\tilde{a}$, when $a, \tau$ and $\tilde{\tau}$ are kept constant. For $\tilde{a}=0.9 a$ there is a loop attached to the origin associated with 4 unstable characteristic exponents. As $\tilde{a} \rightarrow a$, the loop gets smaller and smaller and disappears at $\tilde{a}=a$. If $\tilde{a}$ is just larger than $a$, then the stability diagram turns inside out, the small loop becomes stable and the domain which was stable for $\tilde{a} \leq a$ becomes unstable. This demonstrates that the Smith predictor is sensitive to infinitesimal parameter uncertainties for marginally stable plants.

Figure 6 shows the transition of the stability diagram for a wide range of choices for the estimated system parameter $\tilde{a}$. Arrows show how the D-curves change as a function of a difference between $\tilde{a}$ and $a$. If $\tilde{a}>a$ then the stable domain suddenly reduces to a tiny loop: the size of the loop becomes larger for increasing parameter mismatch. For the extreme case $\tilde{a}=50 a$, the stable domain is close to that of the delayed state feedback controller (denoted by dashed line). The reason for this is that if $\tilde{a} \gg a$, then the value of the predicted state $\tilde{\mathbf{x}}(t)$ can be neglected compared to the actual state $\mathbf{x}(t)$. In this case the Smith predictor is practically equivalent to a delayed state feedback controller. In other words, as the ratio $\tilde{a} / a$ becomes larger, the dynamics of the Smith predictor become those of a delayed state feedback controller. If $\tilde{a}<a$ then the stable domain gets smaller. If $\tilde{a} \leq 0$ then there are no stable domains. Note that for $\tilde{a}=-50 a$, the D-curves approximates the D-curves of the delayed state feedback controller similarly to the case $\tilde{a}=50 a$, but in this case the bounded area is associated with one unstable characteristic exponent.

\section{Sensitivity analysis for an asymptotically stable plant}

In the previous section, it was demonstrated that the Smith predictor is sensitive to infinitesimal parameter mismatches for a marginally stable plant. Dynamic systems in engineering applications often involve damping terms, which results in an asymptotically stable plant. In this section we analyze the same second-order plant with a slight damping term. We consider the system (4)-(6) with

$$
\mathbf{A}=\left(\begin{array}{cc}
0 & 1 \\
-a & -b
\end{array}\right), \quad \tilde{\mathbf{A}}=\left(\begin{array}{cc}
0 & 1 \\
-\tilde{a} & -\tilde{b}
\end{array}\right), \quad \mathbf{B}=\tilde{\mathbf{B}}=\left(\begin{array}{c}
0 \\
-1
\end{array}\right),
$$
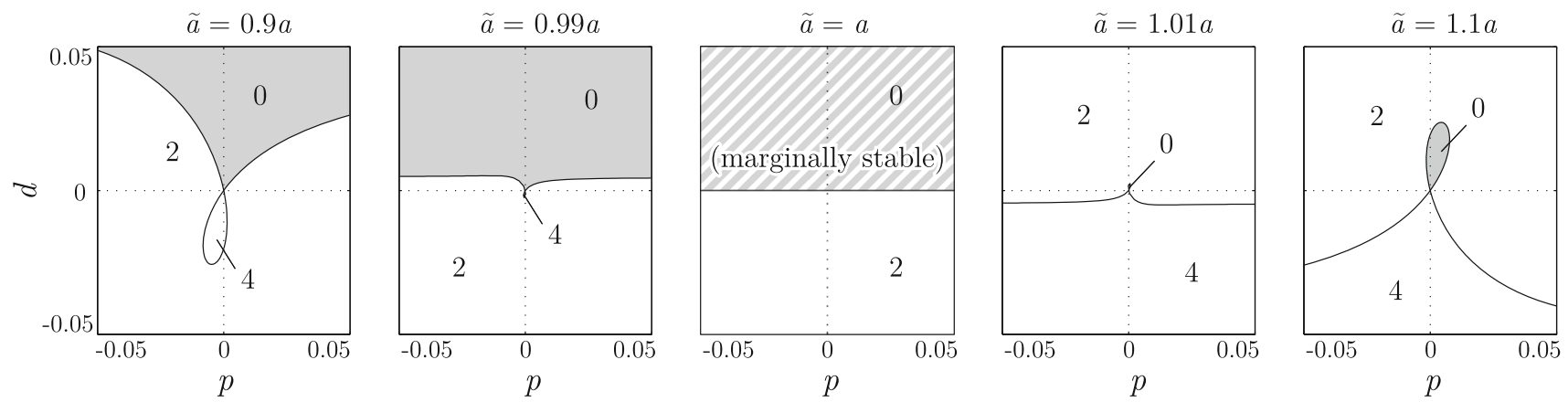

Fig. 5 Transition of the stable domain in case of small parameter mismatch $(a=0.5, \tau=\tilde{\tau}=1)$. Regions of asymptotic stability and marginal stability are indicated by solid gray shading and by striped gray shading, respectively 

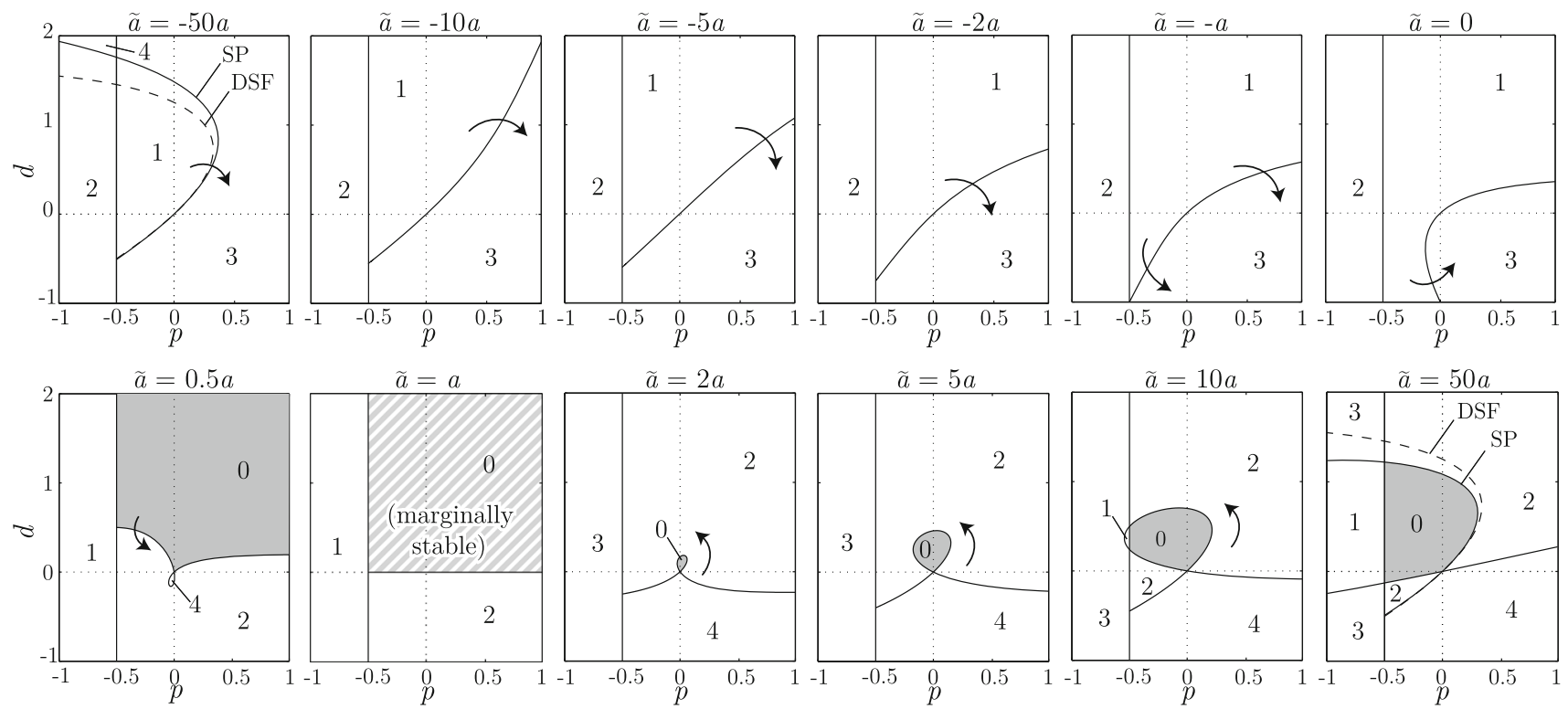

Fig. 6 Stability diagrams with the number of the unstable characteristic exponents for the marginally stable open-loop system with different parameter mismatches $(a=0.5, \tau=\tilde{\tau}=1, D S F$ delayed state feed-

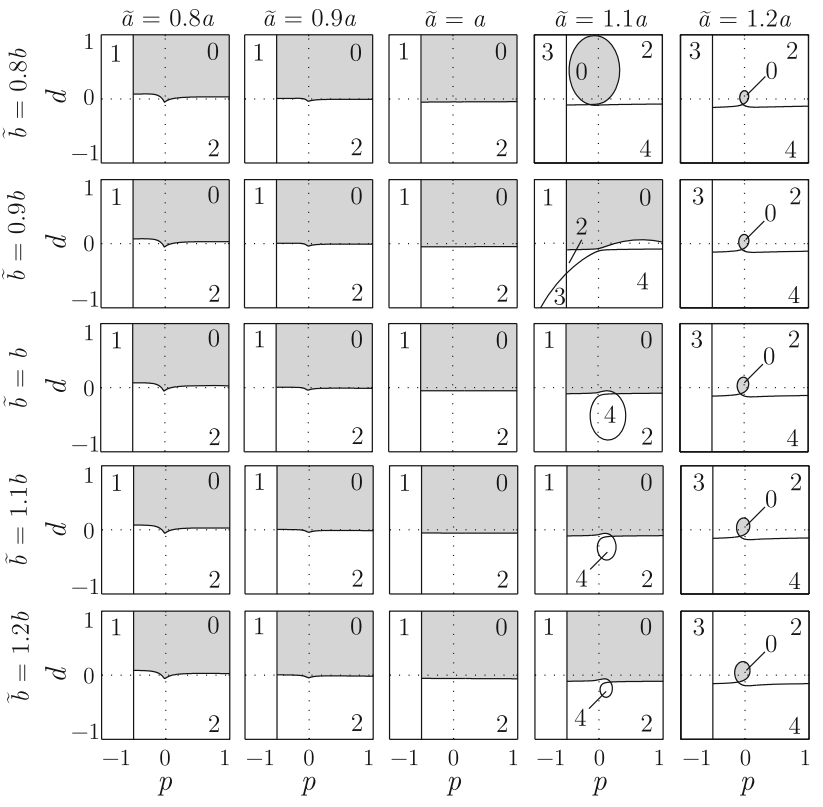

Fig. 7 Stability diagrams with the number of the unstable characteristic exponents for the damped system with small parameter mismatches ( $a=0.5, b=0.05, \tau=\tilde{\tau}=1)$. Regions of asymptotic stability are indicated by solid gray shading

where $b$ is a damping parameter while $\tilde{b}$ is its estimation used by the internal model. Sensitivity of this system is investigated using the same concept as before.

A series of stability diagrams are presented in Fig. 7 for different mismatches of the system parameter and the damping parameter. The damping parameter is $b=0.05$, which back, SP Smith predictor). Regions of asymptotic stability and marginal stability are indicated by solid gray shading and by striped gray shading, respectively

gives the damping ratio of about $3.5 \%$. As opposed to the marginally stable plant, the damped system is not sensitive to infinitesimal parameter mismatches. Still, it is sensitive to finite parameter mismatches: the stable domain shrinks significantly for finite overestimation of the system parameter.

Figure 8 shows the transition of the stability diagram as the estimated system parameter changes. It can be seen that the stable domains shrinks radically while $\tilde{a}$ changes from $1.1 a$ to $1.12 a$. Similarly to the marginally stable plant, the stability diagram tends to that of the delayed state feedback controller (denoted by dashed line) if the system parameter is strongly overestimated.

\section{Stabilization of the unstable plant}

It is a general view that the original Smith predictor (as opposed to its modifications) is capable of compensating the feedback delay for stable plants only. This is true if the plant used by the internal model perfectly matches the real plant, since in this case the poles of the closed-loop system contain the poles of the open-loop system as shown by Eq. (3). However, this argument is not valid for system parameter mismatches.

Figure 9 shows the transition of the stability diagrams for the original Smith predictor described by Eqs. (4)-(6) with (11) for different estimated system parameters in case of an unstable plant with $a=-0.5$ and $\tau=\tilde{\tau}=1$. This figure is the counterpart of Fig. 6 for the unstable plant. The wander- 

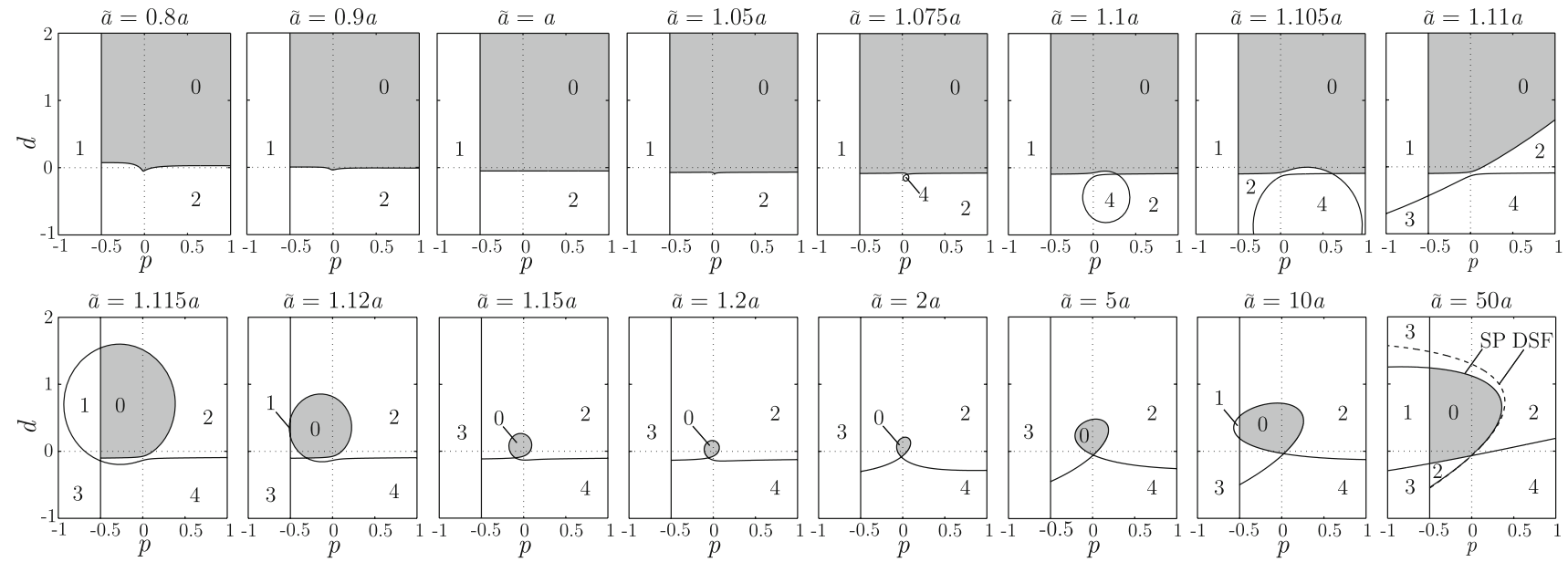

Fig. 8 Stability diagrams with the number of the unstable characteristic exponents for the damped system with different system parameter mismatches ( $a=0.5, b=\tilde{b}=0.05, \tau=\tilde{\tau}=1, D S F$ delayed state feedback, SP Smith predictor). Regions of asymptotic stability are indicated by solid gray shading
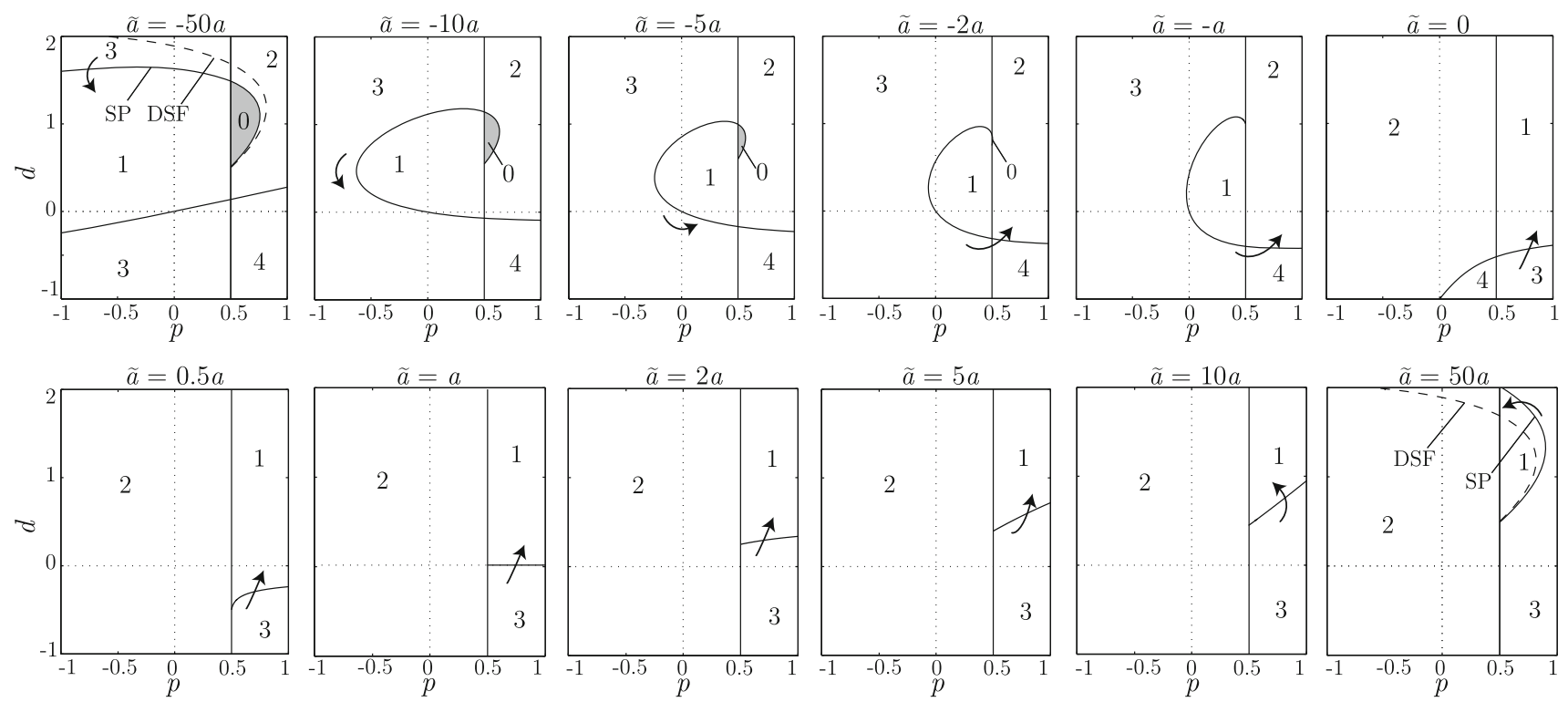

Fig. 9 Stability diagrams with the number of the unstable characteristic exponents for the unstable open-loop system with different parameter mismatches $(a-0.5, \tau=\tilde{\tau}=1, D S F$ delayed state feedback, SP Smith predictor). Regions of asymptotic stability are indicated by gray shading

ing of the D-curves for increasing parameter mismatch can be followed by the arrows in each panel. The number of the unstable characteristic exponents is also presented. For the two extreme cases $\tilde{a}= \pm 50 a$, the D-curves for the corresponding delayed state feedback controller are presented by dashed lines.

As it can be seen, a stable parameter region arises if the system parameter of the internal model is tuned to negative multiples of the actual system parameter (see panels $\tilde{a}=$ $-2 a, \tilde{a}=-5 a, \tilde{a}=-10 a$ and $\tilde{a}=-50 a$ in Fig. 9). In these cases, the unstable plant $(a<0)$ is modeled by a stable system $(\tilde{a}>0)$ ). If $\tilde{a} \rightarrow \infty$ then the stable domain tends to that of the conventional delayed state feedback controller, because, in this case, the predicted state $\tilde{\mathrm{x}}(t)$ can be neglected compared to the actual state $\mathbf{x}(t)$ and the controller behaves like a delayed state-feedback controller. If $\tilde{a}$ gets closer to the actual system parameter then the stable domain disappears and the system cannot be stabilized.

The stable parameter region appears if the tangent of the parametric curve given by Eqs. (18) and (19) at $\omega=0$ is vertical. This implies that stabilization is possible if $\tilde{a}>\tilde{a}_{\text {crit }}$ and $a>a_{\text {crit }}$, where

$$
\tilde{a}_{\text {crit }}=a \frac{\tilde{\tau}\left(a(4 \tau-\tilde{\tau})-\sqrt{-16 a+a^{2}\left(8 \tau^{2}-8 \tau \tilde{\tau}+\tilde{\tau}^{2}\right)}\right)}{4+2 a \tau^{2}}
$$




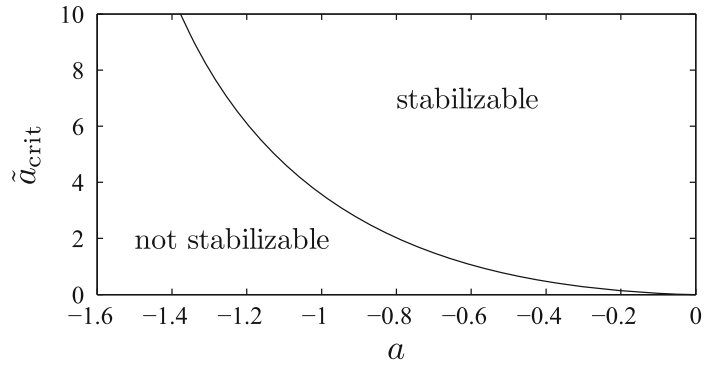

Fig. 10 Stabilizability diagram for $\tau=\tilde{\tau}=1$

is the critical estimated system parameter and $a_{\text {crit }}=-2 / \tau^{2}$ is the critical system parameter in case of delayed state feedback (see Sect. 3.1). Figure 10 presents the limit of stabilizability by the Smith predictor.

Although it can be seen that an extreme tuning of the Smith predictor may stabilize an unstable plant, the real mechanism behind this stabilization is in fact a delayed state feedback. Therefore the practical relevance of this stabilization is limited. Still, this example points out that the general concept that the Smith predictor is not capable to stabilize unstable systems is literally not true.

\section{On the modifications of the Smith predictor}

Since the publication of the original Smith predictor in 1957, several types of its modifications have been developed such as the modified Smith predictor [11,15], the unified Smith predictor [16], the finite spectrum assignment $[18,19]$, the predictive pole placement [12] and the backstepping-based predictor design [10], just to mention a few. These modifications can stabilize unstable systems. It is known that the Smith predictor and the finite spectrum assignment are the predictor-observer and the observer-predictor representation of all stabilizing controllers, respectively $[32,33]$.

Here, we consider the finite spectrum assignment, where prediction is obtained by solving the system equations over the delay period using $\mathbf{x}(t-\tau)$ as initial condition for the prediction. The corresponding control law reads

$\mathbf{u}(t)=\mathbf{K}\left(\mathrm{e}^{\tilde{\mathbf{A}} \tilde{\tau}} \mathbf{x}(t-\tau)+\int_{-\tilde{\tau}}^{0} \mathrm{e}^{-\tilde{\mathbf{A}} \theta} \tilde{\mathbf{B}} \mathbf{u}(\theta+t) \mathrm{d} \theta\right)$.

Here, tilde refers to the estimated parameters in the internal model. It can be seen that the control laws of both the Smith predictor given by Eq. (7) and the finite spectrum assignment given by Eq. (22) involve terms of integrals with respect to the past. There is still a significant difference: while the control law of the Smith predictor involves integral terms over the entire past $[0, t]$, the control law of the finite spectrum assignment employs an integral over the delay period $[t-\tau, t]$ (see also [10]).

It is known that the finite spectrum assignment technique is sensitive to infinitesimal implementation inaccuracies if the integral in the control law (22) is approximated by numerical quadrature rules $[34,35]$. The reason behind this sensitivity is that such an approximation of the integral term results in a neutral functional differential equation, whose strong stability requires the stability of the associated difference equation even if the transfer function of the system is strictly proper. A stabilizability analysis of unstable second-order systems using the finite spectrum assignment in case of parameter mismatches and implementation inaccuracies was presented in [36].

\section{Conclusions}

The Smith predictor was analyzed in the time domain for the second-order system given by Eqs. (4)-(6). It was shown that the closed-loop system can be described by the system of RFDEs (8) and (9). The dimension of the corresponding augmented state vector is double of that of the open-loop system and the equation involves two point delays if the internal model is not perfectly accurate. The corresponding control law involves terms of integrals with respect to the past similarly to the finite spectrum assignment control technique. However, while finite spectrum assignment employs an integral over the delay period $[t-\tau, t]$, the Smith predictor uses an integral over the entire past $[0, t]$.

Sensitivity to parameter mismatches was analyzed for a second-order plant using the D-subdivision method and Stepan's formulas [30]. Particularly, a marginally stable, an asymptotically stable and an unstable plants were considered. It was shown that in case of a marginally stable plant, the closed-loop system is sensitive to the sign of the modeling error. Underestimation of the system parameter does not significantly affect the stability properties, while even the slightest overestimation radically changes the stability diagram. Note that this sensitivity is due to the preservation of the robustness of the plant to parameter uncertainties and not due to the properness of the delay-free transfer function, which was the case in $[20,26]$. A transition between the conventional delayed state feedback and the Smith predictor was illustrated by a series of stability diagrams for a wide range of parameter uncertainties. The same analysis was performed for an asymptotically stable plant. It was shown that he stability of the system was sensitive only to finite parameter mismatches, but not to infinitesimal mismatches.

A stabilizability analysis was performed for an unstable plant. In this case, the system describes the inverted pendulum with feedback delay, which is a paradigm in control theory $[28,29]$, but this model also has a high importance 
in understanding human balancing and human motor control [37-39]. It was shown that the Smith predictor with extremely detuned internal model parameters may stabilize an unstable plant. In this case the predicted state can be neglected compared to the actual state and the stabilization mechanism is practically equivalent to a delayed state feedback.

Acknowledgments This work was supported by the Hungarian National Science Foundation under Grant OTKAK105433. The work reported in the paper has been developed in the framework of the project 'Talent care and cultivation in the scientific workshops of BME' project. This project is supported by the Grant TAMOP-4.2.2.B-10/1-20100009. The authors would like to thank prof. John Milton for his helpful suggestions and comments.

\section{References}

1. Kidd M, Stepan G (2014) Delayed control of an elastic beam. Int J Dyn Control 2(1):68-76

2. Lehotzky D, Insperger T (2012) Stability of turning processes subjected to digital pd control. Mech Eng 56(1):33-42

3. Wang ZH, Hu HY (2006) Stabilization of vibration systems via delayed state difference feedback. J Sound Vib 296(1-2):117-129

4. Wei F, Bachrathy D, Orosz G, Ulsoy AG (2014) Spectrum design using distributed delay. Int J Dyn Control 2(2):234-246

5. Orosz G, Stepan G (2006) Subcritical Hopf bifurcations in a car-following model with reaction- time delay. Proc R Soc A 462(2073):2643-2670

6. Erneux T, Kalmar-Nagy T (2007) Nonlinear stability of a delayed feedback controlled container crane. J Vib Control 13(5):603-616

7. Ma H, Butcher EA (2005) Stability of elastic columns with periodic retarded follower forces. J Sound Vib 286:849-867

8. Cepeda-Gomez R, Olgac N (2014) A consensus protocol under directed communications with two time delays and delay scheduling. Int J Control 87(2):291-300

9. Smith OJM (1957) Closer control of loops with dead time. Chem Eng Prog 53(5):217-219

10. Krstic M (2009) Delay compensation for nonlinear, adaptive, and PDE systems. Birkhauser, Boston

11. Astrom KJ, Hang CC, Lim BC (1994) A new Smith predictor for controlling a process with an integrator and long dead-time. IEEE Trans Autom Control 39(2):343-345

12. Gawthrop PJ, Ronco E (2002) Predictive pole-placement control with linear models. Automatica 38(3):421-432

13. Jankovic M (2009) Forwarding, backstepping, and finite spectrum assignment for time delay systems. Automatica 45(1):2-9

14. Michiels W, Roose D (2001) Time delay compensation in unstable plants using delayed state feedback. In: Proceeding IEEE Conference on Decision and Control, 1433-1437 December 2001

15. Palmor ZJ (2000) Time-delay compensation Smith predictor and its modifications. In: Levine W (ed) The Control Handbook. CRC Press, Boca Raton

16. Zhong QC, Weiss G (2004) A unified smith predictor based on the spectral decomposition of the plant. Int J Control 77(15):13621371
17. Krstic M, Bekiaris-Liberis N (2010) Compensation of infinitedimensional input dynamics. Annu Rev Control 34(2):233-244

18. Manitius AZ, Olbrot AW (1979) Finite spectrum assignment problem for systems with delays. IEEE Trans Autom Control AC24:541-553

19. Wang QG, Lee TH, Tan KK (1999) Finite spectrum assignment for time delay systems. Springer, London

20. Michiels W, Niculescu SI (2003) On the delay sensitivity of Smith predictors. Int J Syst Sci 34(8-9):543-551

21. Michiels W, Niculescu SI (2007) Stability and stabilization of timedelay systems: an eigenvalue-based approach. SIAM Publications, Philadelphia

22. Palmor ZJ (1980) Stability properties of Smith dead-time compensator controllers. Int J Control 32:937-949

23. Feng W (1991) On practical stability of linear multivariable feedback systems with time-delays. Automatica 27(2):389-394

24. Owens DH, Raya A (1982) Robust stability of Smith predictor controllers for time-delay systems. IEE Proc D 129(6):298-304

25. Yamanaka K, Shimemura E (2012) Effects of mismatched Smith controller on stability in systems with time-delay. Automatica 23(6):787-791

26. Gu K (2012) A review of some subtleties of practical relevance for time-delay systems of neutral type. ISRN Appl. Math. 2012, 46 p. doi: $10.5402 / 2012 / 725783$

27. Insperger T, Milton J, Stepan G (2013) Acceleration feedback improves balancing against reflex delay. J R Soc Interface 10(79):1742-5662

28. Qin ZC, Li X, Zhong S, Sun JQ (2014) Control experiments on time-delayed dynamical systems. J. Vib. Control. doi:10.1177/ 1077546312469424

29. Sieber J, Krauskopf B (2005) Extending the permissible control loop latency for the controlled inverted pendulum. Dyn Syst 20(2):189-199

30. Stepan G (1989) Retarded dynamical systems. Longman, Harlow

31. Morari M, Zafiriou E (1989) Robust process control. Prentice Hall, Englewood Cliffs

32. Mirkin L, Raskin N (2003) Every stabilizing dead-time controller has an observerpredictor-based structure. Automatica 39:17471754

33. Zhong QC (2006) Robust control of time-delay systems. Springer, London

34. Michiels W, Mondie S, Roose D (2003) Robust stabilization of time-delay systems with distributed delay control laws: Necessary and sufficient conditions for a safe implementation. Technical report, TWReport 363, Department of Computer Science, Katholieke Universiteit Leuven, Leuven

35. Mondie S, Dambrine M, Santos O (2002) Approximation of control laws with distributed delays: a necessary condition for stability. Kybernetika 38(5):541-551

36. Molnar TG, Insperger T (2014) On the robust stabilizability of unstable systems with feedback delay by finite spectrum assignment. J. Vib. Control. doi:10.1177/1077546314529602

37. Loram ID, Lakie M (2002) Human balancing of an inverted pendulum: position control by small, ballistic-like, throw and catch movements. J Physiol 540(3):1111-1124

38. Milton J, Cabrera JL, Ohira T, Tajima S, Tonosaki Y, Eurich CW, Campbell SA (2009) The time-delayed inverted pendulum: Implications for human balance control. Chaos doi:10.1063/1.3141429

39. Stepan G (2009) Delay effects in the human sensory system during balancing. Philos Trans R Soc A 367:1195-1212 